\title{
Bargaining and the MISO Interference Channel
}

\author{
Matthew Nokleby' ${ }^{1}$ and A. Lee Swindlehurst ${ }^{2}$ \\ ${ }^{1}$ Department of Electrical and Computer Engineering, Rice University, Houston, TX 77005, USA \\ ${ }^{2}$ Department of Electrical Engineering and Computer Science, University of California at Irvine, Irvine, \\ CA 92697, USA \\ Correspondence should be addressed to Matthew Nokleby, nokleby@rice.edu
}

Received 31 October 2008; Revised 27 February 2009; Accepted 8 April 2009

Recommended by Eduard A. Jorswieck

\begin{abstract}
We examine the MISO interference channel under cooperative bargaining theory. Bargaining approaches such as the Nash and Kalai-Smorodinsky solutions have previously been used in wireless networks to strike a balance between max-sum efficiency and max-min equity in users' rates. However, cooperative bargaining for the MISO interference channel has only been studied extensively for the two-user case. We present an algorithm that finds the optimal Kalai-Smorodinsky beamformers for an arbitrary number of users. We also consider joint scheduling and beamformer selection, using gradient ascent to find a stationary point of the Kalai-Smorodinsky objective function. When interference is strong, the flexibility allowed by scheduling compensates for the performance loss due to local optimization. Finally, we explore the benefits of power control, showing that power control provides nontrivial throughput gains when the number of transmitter/receiver pairs is greater than the number of transmit antennas.
\end{abstract}

Copyright ( $) 2009$ M. Nokleby and A. L. Swindlehurst. This is an open access article distributed under the Creative Commons Attribution License, which permits unrestricted use, distribution, and reproduction in any medium, provided the original work is properly cited.

\section{Introduction}

After more than a decade of intense research, multiantenna communications systems are sufficiently well understood that they now appear in current and emerging wireless standards $[1,2]$. Because they offer increased spatial flexibility, multiple-antenna systems are particularly well suited to multiuser communications. Generally speaking, multiuser communication presents a complicated problem partially because performance criteria are difficult to characterize. There is no, for example, single data rate or bit-error probability to optimize. Instead, we can only maximize composite performance measures such as the network sum rate, maxmin fairness, or quality-of-service requirements. Ultimately, the choice of objective function is often somewhat arbitrary.

To meet this challenge, researchers have begun to apply game theory [3], a mathematical idealization of human decision-making, to problems in multiuser communications. Game theory provides a systematic framework for the study of decision makers with potentially conflicting interests, as well as solutions for such conflicts. Accordingly, a game-theoretic analysis can provide a tractable, structured approach to resource allocation. Researchers have successfully employed game-theoretic ideas to design "fair" medium-access protocols, develop decentralized network algorithms, and otherwise solve resource-allocation problems in communications networks [4-10].

In this paper, we study the multiple-input single-output (MISO) interference channel. In the MISO interference channel, several communication links, each involving a multiantenna transmitter and a single-antenna receiver, are simultaneously active. This scenario models, for example, intercell interference in cellular systems or MIMO networks where receivers employ fixed beamformers. Multilink MISO systems have been studied in a number of previous works. For example, in $[11,12]$, the MISO broadcast channel is studied from the intercell interference point of view, with emphasis on maximizing the network sum rate. The same scenario is addressed in [13], but max-min fairness is used to improve the performance of weaker network links. Gametheoretic solutions for the MISO interference channel based on bargaining have been considered in $[14,15]$, but only for the two-user case.

Our particular focus is to maximize network performance according to the Kalai-Smorodinsky solution [16], 
a cooperative bargaining approach closely related to the well-known Nash bargaining solution [17]. For our problem, the fundamental idea of the Kalai-Smorodinsky (K$S)$ approach is to maximize users' rates while ensuring that users experience the same fraction of the rate they would achieve without interference. In practice, the K-S solution defines a compromise between efficiency (defined herein in terms of maximizing the sum rate) and equity (maximizing the minimum rate). Our primary contribution is an algorithm that efficiently finds the K-S solution for an arbitrary number of users, rather than just the twouser case. We transform the rate-maximization problem to a series of convex programming problems, allowing us to find the beamformers that achieve the rates defined by the K-S solution.

A drawback of the K-S solution is that when interference becomes strong for a single user, all users' bargained rates tend toward zero. To avoid this, we also study joint scheduling and beamformer selection under K-S bargaining, which introduces a temporal degree of freedom for avoiding interference. Scheduling also convexifies the feasible rate region, which is an important consideration in cooperative bargaining. However, the need to jointly address scheduling and beamformer selection complicates the optimization, preventing us from easily finding the K-S solution. We therefore devise a gradient-based algorithm to find a stationary point of the K-S objective function. While we sacrifice global optimality to include scheduling, the performance advantage of employing time-division multiplexing significantly outweighs the potential loss of optimality when the interference is strong.

The paper is organized as follows: in Section 2 we present the system model, discussing the achievable rates and a few simple beamforming strategies. In Section 3 we briefly introduce the Kalai-Smorodinsky solution. In Section 4 we present algorithms for selecting beamformers and (where applicable) transmission schedules that achieve the KalaiSmorodinsky solution. In Section 5 we examine the fairness and efficiency of our proposed algorithms and discuss the effects of power control. Finally, we give our conclusions in Section 6.

\section{System Model}

2.1. Signal Model. The $K$-user MISO interference channel, as depicted in Figure 1, is composed of $K N$-antenna transmitters, each of which intends to communicate with a unique single-antenna receiver. We assume a narrowband channel model where the $i$ th transmitter sends a complex baseband vector $\mathbf{x}_{i}$. The received signal $y_{i}$ contains the intended signal, cochannel interference from the other $K-1$ transmitters, and additive Gaussian noise:

$$
y_{i}=\mathbf{h}_{i, i}^{H} \mathbf{x}_{i}+\sum_{\substack{j=1 \\ j \neq i}}^{K} \mathbf{h}_{i, j}^{H} \mathbf{x}_{j}+n_{i},
$$

where $\mathbf{h}_{i, j}$ is the vector of complex channel gains between the antennas of the $j$ th transmitter and the $i$ th receiver, and

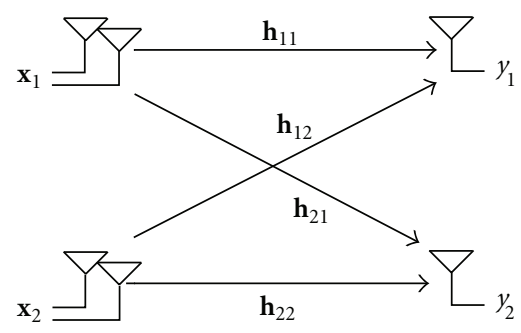

FIgURE 1: Two-user MISO interference channel.

$(\cdot)^{H}$ denotes the Hermitian transpose. We normalize the channel gains such that - without loss of generality- $n_{i}$ has unit variance. Particularly, we assume channels of the form $\mathbf{h}_{i, j}=\sqrt{\rho_{i, j}} \hat{\mathbf{h}}_{i, j}$, where the elements of $\hat{\mathbf{h}}_{i, j}$ are zero-mean, unit-variance complex random variables, and $\rho_{i, j}$ represents the expected channel gain between the $j$ th transmitter and the $i$ th receiver.

2.2. Achievable Rates. To define the set of achievable rates under our assumptions, we view each transmitted signal $\mathbf{x}_{i}$ as a zero-mean random vector characterized by the covariance matrix $\mathbf{P}_{i}=E\left\{\mathbf{x}_{i} \mathbf{x}_{i}^{H}\right\}$, where $E\{\cdot\}$ denotes statistical expectation. In principle, $\mathbf{P}_{i}$ can be any positive semidefinite matrix, although we focus on the rank-one case due to the MISO setting considered here. Specifically, we assume that $\mathbf{x}_{i}$ is of the form $\mathbf{x}_{i}=\mathbf{w}_{i} s_{i}$, where $\mathbf{w}_{i}$ is the (fixed) transmit beamformer for user $i$, and $s_{i}$ is a zero-mean, unit-variance Gaussian symbol. Thus, $\mathbf{P}_{i}=\mathbf{w}_{i} \mathbf{w}_{i}^{H}$, and the spatial characteristics of the transmitted signal are entirely characterized by the beamforming vector $\mathbf{w}_{i}$.

Each transmitter has limited peak power output, which we model by constraining the norm of each beamformer: $\left\|\mathbf{w}_{i}\right\|_{2} \leq 1$, where $\|\cdot\|_{2}$ denotes the $\ell_{2}$ norm. Let $\mathfrak{W}_{1}$ denote the set of feasible beamformers:

$$
\mathbb{W}_{1}=\left\{\mathbf{w} \in \mathbb{C}^{N}:\|\mathbf{w}\|_{2} \leq 1\right\} .
$$

Here we have defined a general model where transmitters choose both the magnitude of the beamformer, which represents the transmit power, as well as its direction. When the beamformers are unit-norm, the channel parameter $\rho_{i, j}$ represents the received signal-to-noise ratio (SNR) between the $j$ th transmitter and the $i$ th receiver. We may wonder, given the spatial freedom offered by the multiple antennas, if such power control is necessary. For example, in [18] it is shown that, when $K \leq N$, only beamformers with $\left\|\mathbf{w}_{i}\right\|_{2}=1$ are necessary, obviating the need for power control. However, this result does not generalize, and in Section 5 we explore the benefit of power control in a system with an arbitrary number of users.

In determining achievable rates, we assume that transmitters and receivers have full channel state information and that the receivers employ single-user detection, meaning that cochannel interference is treated as noise when decoding the incoming signal. Under these assumptions, the rate across 
the $i$ th link is bounded by the mutual information between $\mathbf{x}_{i}$ and $y_{i}$, which, in terms of the beamformers, is

$$
I\left(\mathbf{x}_{i} ; y_{i}\right)=\log _{2}\left(1+\frac{\left|\mathbf{h}_{i, i}^{H} \mathbf{w}_{i}\right|^{2}}{1+\sum_{j \neq i}\left|\mathbf{h}_{i, j}^{H} \mathbf{w}_{j}\right|^{2}}\right) .
$$

For notational convenience we will occasionally group the beamformers into a single $N \times K$ matrix $\mathbf{W}=$ $\left[\begin{array}{llll}\mathbf{w}_{1} & \mathbf{w}_{2} & \cdots & \mathbf{w}_{K}\end{array}\right]$. Then, we can denote the mutual information across the $i$ th link as a function of the beamformers: $I_{i}(\mathbf{W})$. The set of achievable rates is bounded by the mutual information possible under all feasible beamformers:

$$
\mathcal{R}=\left\{\mathbf{r} \in \mathbb{R}_{+}^{K}: r_{i} \leq I_{i}(\mathbf{W}), \mathbf{W} \in \mathcal{W}_{1}^{K}\right\} .
$$

The feasible set $\mathcal{R}$ has an important property which we will exploit throughout the paper: it is comprehensive with respect to the zero vector. A set $\delta \subset \mathbb{B}^{K}$ is comprehensive with respect to 0 provided that for every $\mathbf{r} \in \delta, 0 \preceq \mathbf{s} \preceq \mathbf{r}$ implies $\mathbf{s} \in \mathcal{8}$, where $\preceq$ and $\succeq$ represent element-wise vector inequalities. In our case, the rate region $\mathcal{R}$ is comprehensive because any user can-without altering its beamformerfreely lower its rate without impacting other users' rates.

2.3. Scheduling. In general, $\mathcal{R}$ is not convex, suggesting that we may achieve higher rates-especially in cases of strong interference-via time-sharing. (Alternatively, the rate region may be convexified by other equivalent means such as frequency-sharing or randomized beamformer selection). To do so, we divide each transmission into $K$ time blocks, during each of which the transmitters may use a different beamformer. The mutual information during block $t$ is

$$
I\left(\mathbf{x}_{i}(t) ; y_{i}(t)\right)=\log _{2}\left(1+\frac{\left|\mathbf{h}_{i, i}^{H} \mathbf{w}_{i}(t)\right|^{2}}{1+\sum_{j \neq i}\left|\mathbf{h}_{i, j}^{H} \mathbf{w}_{j}(t)\right|^{2}}\right) .
$$

We use $I_{i}(\mathbf{W}(t))$ to represent the mutual information during the $t$ th block.

The relative duration of each block is defined by the scheduling vector $\mathbf{a}=\left[\begin{array}{lll}a_{1} & \cdots & a_{K}\end{array}\right]$, which obeys the constraints $\mathbf{a} \geq 0$ and $\sum_{t=1}^{K} a_{t}=1$. The scheduling weights in a define a convex combination of the rates achieved during each time block. With scheduling, the average achievable rate over the $i$ th link is bounded by the average mutual information $\sum_{t} a_{t} I\left(\mathbf{x}_{i}(t) ; \mathbf{y}(t)\right)$. The set of feasible scheduling vectors is

$$
\mathcal{A}=\left\{\mathbf{a} \in \mathbb{R}_{+}^{K}: \sum_{t=1}^{K} a_{t}=1\right\} .
$$

Since time-sharing allows us to take convex combinations of rate vectors, the set of achievable rates under scheduling, denoted by $\overline{\mathcal{R}}$, is the convex hull of $\mathcal{R}$ :

$$
\overline{\mathcal{R}}=\left\{\mathbf{r} \in \mathbb{R}_{+}^{K}: r_{i} \leq \sum_{t=1}^{K} a_{t} I_{i}(\mathbf{W}(t)), \mathbf{a} \in \mathcal{A}, \mathbf{W}(t) \in \mathfrak{W}_{1}^{K}, \forall t\right\} .
$$

To see that $K$ time blocks are sufficient to achieve the convex hull, note that the convex hull of $\mathcal{R}$ can be defined as the intersection of all closed half-planes in $\mathbb{R}^{K}$ that contain $\mathcal{R}$. So, any boundary point on the convex hull of $\mathcal{R}$ must lie on a convex subset of a bounding hyperplane in $\mathbb{R}^{K}$ defined by at most $K$ linearly independent boundary points of $\mathcal{R}$. Thus any point on the boundary of the convex hull can be achieved by taking convex combinations of at most $K$ points in $\mathcal{R}$. But, since $\mathcal{R}$ is comprehensive, we can reach any point in the convex hull by choosing the nearest boundary point and appropriately lowering the rates of the associated $K$ points. To see that $K$ points are required in general, consider an extreme case where $\rho_{i, j}=\infty$ for $i \neq j$, so only a single transmitter can achieve a nonzero rate at a time. To realize the boundary of the convex hull of $\mathcal{R}$, each user needs its own block in which to transmit, necessitating $K$ blocks.

2.4. Beamforming Strategies. A few simple strategies for choosing beamformers have previously been proposed. The first is the Nash equilibrium (NE) beamformer [14], where each transmitter maximizes its own mutual information without regard for others. The NE beamformer relies on the fact that, regardless of interference, a transmitter maximizes its mutual information simply by maximizing $\left|\mathbf{h}_{i, i}^{H} \mathbf{w}_{i}\right|^{2}$. By the Cauchy-Schwarz inequality, the NE beamformer is

$$
\mathbf{w}_{i}^{\mathrm{NE}}=\frac{\mathbf{h}_{i, i}}{\left\|\mathbf{h}_{i, i}\right\|_{2}} .
$$

In game-theoretic terms, this choice of beamformers is a Nash equilibrium [19], meaning that no single transmitter can improve its rate by switching to a different beamformer. While the Nash equilibrium is individually optimal from each user's perspective, it is frequently possible for transmitters to jointly choose beamformers such that each user's rate is higher than the NE rate. Indeed, the NE has notoriously poor performance, especially when interference is strong.

The zero-forcing strategy [14] takes the opposite approach, focusing entirely on eliminating cochannel interference in order to maximize the mutual information of other users. To specify this beamformer, let $\mathbf{H}_{-i}$ be the $N \times$ $K-1$ matrix containing all of the interference channels for the $i$ th transmitter:

$$
\mathbf{H}_{-i}=\left[\begin{array}{llllll}
\mathbf{h}_{1, i} & \cdots & \mathbf{h}_{i-1, i} & \mathbf{h}_{i+1, i} & \cdots & \mathbf{h}_{K, i}
\end{array}\right] .
$$

Then, we get the zero-forcing beamformer $\mathbf{w}_{i}^{\mathrm{ZF}}$ by projecting the NE beamformer onto the orthogonal complement of the column space of $\mathbf{H}_{-i}$ :

$$
\mathbf{w}_{i}^{\mathrm{ZF}}=\frac{\Pi_{\mathbf{H}_{-i}}^{\perp} \mathbf{h}_{i, i}}{\left\|\Pi_{\mathbf{H}_{-i}}^{\perp} \mathbf{h}_{i, i}\right\|_{2}},
$$

where $\Pi_{\mathbf{H}_{-i}}^{\perp}$ represents the appropriate orthogonal projection. By choosing $\mathbf{w}_{i}^{\mathrm{ZF}}$, a transmitter maximizes the mutual information across the ith channel after ensuring that its signal creates no cochannel interference. However, for randomly generated channels, $\mathbf{w}_{i}^{\mathrm{ZF}}=0$ almost surely when $K>N$. In such cases, zero-forcing trivially eliminates 
interference by choosing the zero vector unless $\mathbf{h}_{i, i}$ is outside of the column space of $\mathbf{H}_{-i}$, which occurs with probability zero.

Finally, we can also eliminate interference via simple time-division multiple access (TDMA) scheduling. We divide up the transmission into equally spaced blocks by setting $a_{t}=1 / K$ for every $t$, and we allow each transmitter to signal, without interference, during a single block:

$$
\mathbf{w}_{i}^{\mathrm{TDMA}}(t)= \begin{cases}\frac{\mathbf{h}_{i, i}}{\left\|\mathbf{h}_{i, i}\right\|_{2}}, & \text { if } t=i, \\ 0, & \text { otherwise. }\end{cases}
$$

TDMA guarantees that each user has a nonzero rate regardless of interference strength as long as $K$ is finite. However, this approach entirely ignores the possibility of interference mitigation through beamforming. So, to select beamformers more comprehensively, we must clearly define our desired performance criteria, which we discuss in the next section.

\section{Kalai-Smorodinsky Solution}

We briefly introduce the Kalai-Smorodinsky (K-S) solution in an abstract setting, which we then apply to the MISO interference channel. A $K$-player bargaining game is formally defined by a set of feasible payoffs $u \subset \mathbb{R}^{K}$ and a disagreement point $\delta \in \mathcal{U}$. The disagreement point represents the utility guaranteed to each player should bargaining fail. In bargaining games, players cooperatively choose a compromise point. That is, rather than myopically maximizing individual payoff, players jointly choose a strategy that results in a mutually agreeable payoff vector. A bargaining solution is a mapping $f(U, \boldsymbol{\delta})$ to a payoff vector $\mathbf{u}^{*} \in \mathcal{U}$ such that $\mathbf{u}^{*} \succeq \boldsymbol{\delta}$.

The K-S solution is an axiomatic bargaining solution, meaning that it is characterized abstractly by a set of (ostensibly) reasonable axioms rather than by a concrete bargaining process. First, define the ideal point $\mathbf{b}(\mathcal{U}, \boldsymbol{\delta})$ element-wise by

$$
b_{i}(\mathcal{U}, \boldsymbol{\delta})=\max \left\{u_{i}: \mathbf{u} \in \mathcal{U}, \mathbf{u} \succeq \boldsymbol{\delta}\right\} .
$$

The ideal point $\mathbf{b}$ expresses the best-case utility for each player. Then, the K-S solution is defined by the following axioms.

(1) Pareto Efficiency. If $\mathbf{u} \in \mathcal{U}$ is a vector such that $\mathbf{u} \succeq \mathbf{u}^{*}$, then $\mathbf{u}=\mathbf{u}^{*}$. That is, there is no point $\mathbf{u} \in \mathcal{U}$ such that any player receives higher payoff than under $\mathbf{u}^{*}$ without penalizing another player. If there is a player $i$ for which $u_{i}>u_{i}^{*}$, then there must be at least one player $j$ for which $u_{j}<u_{j}^{*}$. Pareto efficiency ensures that we do not overlook any points which improve players' payoff without cost to other players.

(2) Invariance to Positive Affine Transformations. Let $A$ be a positive affine transformation; that is, $l(\mathbf{s})=\left(c_{1} s_{1}+\right.$ $\left.d_{1}, \ldots, c_{K} s_{K}+d_{K}\right)^{T}$ for positive $c_{i}$ and arbitrary $d_{i}$. Then, if $f(U, \delta)=\mathbf{u}^{*}$, then $f(l(\mathcal{U}), l(\boldsymbol{\delta}))=l\left(\mathbf{u}^{*}\right)$. In short, the solution must be independent to the scale and zero level of the players' utilities.

(3) Symmetry. Let $T$ be a permutation of the players. Then, $f(T(\mathcal{U}, T(\delta)))=T\left(\mathbf{u}^{*}\right)$ whenever $f(\boldsymbol{U}, \boldsymbol{\delta})$. Here we impose a minimal sense of fairness on the solution. Since players may be interchanged without effect, each player obtains equal utility $\left(u_{i}^{*}=u_{j}^{*}\right.$, for all $\left.i, j\right)$ if $U$ is symmetric and $\delta_{i}=\delta_{j}$, for all $i, j$.

(4) Monotonicity. Let $(\mathcal{U}, \boldsymbol{\delta})$ and $(\mathcal{V}, \boldsymbol{\delta})$ be bargaining games such that $\mathcal{V} \supseteq \mathcal{U}$ and $\mathbf{b}(\mathcal{U}, \boldsymbol{\delta})=\mathbf{b}(\mathcal{V}, \boldsymbol{\delta})$. Then, $f(\mathcal{V}, \boldsymbol{\delta}) \succeq$ $f(\mathcal{U}, \boldsymbol{\delta})$.

While Axioms 1 and 3 seem obvious for a fair and efficient bargain, Axioms 2 and 4 merit further discussion in the context of bargaining in a wireless network. Invariance to affine transformations is usually invoked because the scale (or the units) of players' utilities may be different. The so-called interpersonal comparison of utilities is therefore undesirable, since the utilities are incommensurable. Axiom 2 solves the commensurability problem by making the solution independent to the scale level of players' utilities; the units are abstracted away by the bargaining solution.

For our problem, we have expressed each player's utility function in the same units (bits/sec/Hz), perhaps suggesting that Axiom 2 is unnecessary. While there is much to be said about the appropriateness of affine invariance, we note the following practical observation: different users may regard equal rates differently. A user with lower quality-of-service demands, for example, might assign higher utility to a particular rate than would a user with higher demands. So, users' true utilities are arbitrary (but presumably nondecreasing) functions of the rates. In identifying the users' utilities as the rates and invoking affine invariance, we tacitly assume that the true utilities are positive affine functions of the rates, with scale and zero level unknown. In this case, invariance to positive affine transformations is a necessary criterion for bargaining among the wireless users.

Axiom 4 prescribes a subjective notion of fairness by dictating the variation of the solution under changes in $u$. Monotonicity ensures that if we expand the set of feasible utilities, the bargained utility to each player can only increase. Indirectly, monotonicity ensures that stronger players receive higher payoff and are not unduly penalized by bargaining.

In its original presentation [16], it is shown that a unique solution $f(\boldsymbol{U}, \boldsymbol{\delta})$ satisfies Axioms 1-4 for any twoplayer game in which $U$ is both compact and convex. In order to generalize the solution to $K$ players, however, we need to place further restrictions on $U$ [22]. Fortunately, the generalization is straightforward when we restrict our attention to the class of bargaining games where $u$ is also comprehensive $[20,23]$ and satisfies the following property: if $\mathbf{u}, \mathbf{v} \in \mathcal{U}$ satsify $\mathbf{u} \neq \mathbf{v}$ and $\mathbf{u} \preceq \mathbf{v}$, then there exists $\mathbf{w} \in \mathcal{U}$ such that $\mathbf{w}$ strictly dominates $\mathbf{u}$, or $\mathbf{w} \succ \mathbf{u}$.

As long as $\mathcal{U}$ is compact, convex, comprehensive, and satisfies the above criterion, the four axioms lead to a unique solution with a convenient geometric interpretation, as depicted in Figure 2 for $\boldsymbol{\delta}=0$. The K-S solution $\mathbf{u}^{*}$ is 
the largest element in $U$ (with respect to any norm) that lies along the line segment connecting $\boldsymbol{\delta}$ with $\mathbf{b}$, or the maximum point $\mathbf{u}$ such that $\left(u_{i}-\delta_{i}\right) /\left(b_{i}-\delta_{i}\right)=\left(u_{j}-\delta_{j}\right) /\left(b_{j}-\delta_{j}\right)$ for all $i, j$. Equivalently, we can express the K-S solution as an optimization over a weighted minimum objective function:

$$
\mathbf{u}^{*}=\arg \max _{\mathbf{u} \in \mathcal{U}} \min \left(\frac{u_{1}-\delta_{1}}{b_{1}-\delta_{1}}, \ldots, \frac{u_{K}-\delta_{K}}{b_{K}-\delta_{K}}\right) .
$$

The solution (13) exposes a connection between the K-S solution and max-min fairness, which focuses on improving the payoff of the weakest players. While max-min is a widely accepted criterion of fairness in both human and artificial systems [24-27], it allows weak players to limit (unfairly, one might argue) the payoff of stronger players, especially when $\mathcal{U}$ is highly asymmetric $[28,29]$. "Fairness" is ultimately a subjective notion, so we refer to the max-min payoffs as equitable rather than fair, since max-min gives equal payoff to all users for convex $\mathcal{U}$.

Rather than strictly maximizing the minimum rate, the $\mathrm{K}-\mathrm{S}$ solution normalizes the payoffs according to the shape of $U$, placing a premium on increasing payoff to players with higher best-case payoff $b_{i}$. Doing so increases the sum payoff at the cost of the payoff of the weakest player. In practice, we may regard the K-S solution as a balance between strict max-min equity and max-sum efficiency, a position further justified by the results in Section 5 .

3.1. Convexity. Of course, since the achievable rates for the MISO interference channel are only convex under scheduling, we should also consider K-S bargaining when $U$ is not convex. Fortunately, the K-S solution has also been studied for nonconvex $\mathcal{U}[30,31]$. It is shown in [30] that by weakening Pareto efficiency, the solution given above extends to comprehensive, compact, but nonconvex $U$. Specifically, Pareto efficiency is replaced with the following axiom.

(5) Weak Pareto Efficiency. If $\mathbf{u} \in \mathcal{U}$ is a vector such that $\mathbf{u} \succ \mathbf{u}^{*}$, then $\mathbf{u}=\mathbf{u}^{*}$. That is, there exists no other $\mathbf{u} \in \mathcal{U}$ such that every player obtains higher payoff than in $\mathbf{u}^{*}$. In contrast to strong Pareto efficiency, it may indeed be possible to find a point $\mathbf{u} \in \mathcal{U}$ that improves several players' utilities without harming other players.

As long as $U$ is compact and comprehensive, the maximal element in $U$ along the line segment connecting $\boldsymbol{\delta}$ and $\mathbf{b}$ is the unique solution satisfying Axioms 2-5. Since $\mathcal{U}$ is nonconvex, the solution point $\mathbf{u}^{*}$ may not be the unique weighted max-min point from (13), since there may be multiple max-min points as depicted in Figure 3. If, of course, the weak Pareto frontier of $U$ coincides with its strong Pareto frontier, $\mathbf{u}^{*}$ is still Pareto efficient and corresponds to the unique weighted max-min point as before. As we will see in Section 5.2, this is usually the case with the rate regions associated with the MISO interference channel.

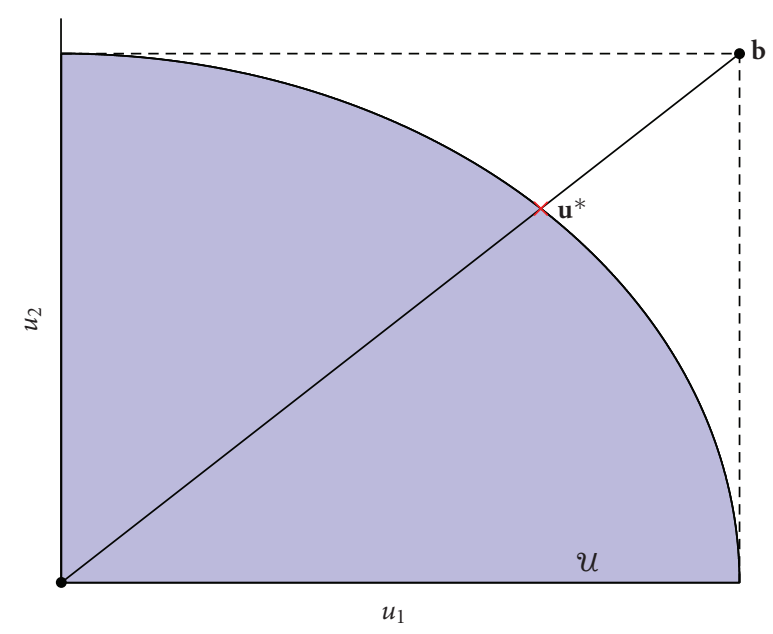

FIGURE 2: K-S solution for a convex payoff set.

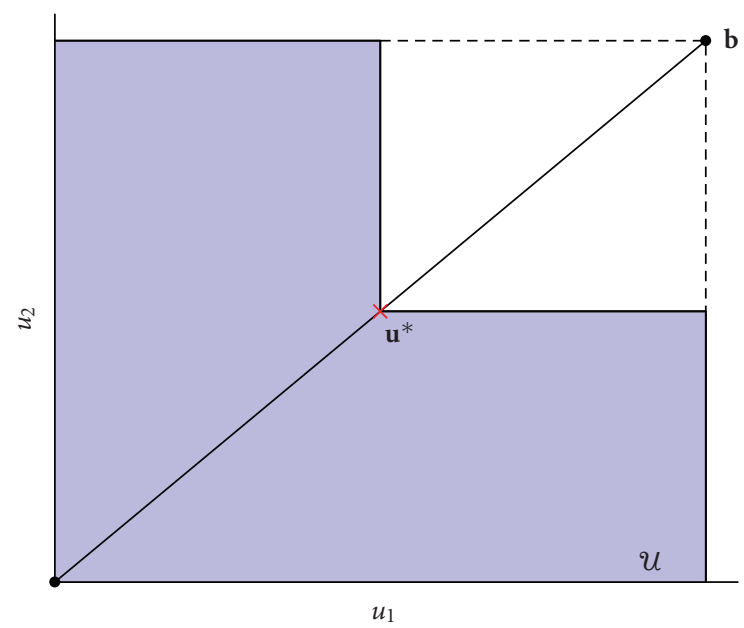

FIGURE 3: K-S solution for a nonconvex payoff set. Note that, in this case, the solution point is only weakly Pareto efficient.

\section{K-S Bargaining for the MISO Channel}

Finding the K-S solution for the MISO interference channel requires that we cast the problem in the game-theoretic framework discussed in the previous section. The recasting is straightforward. The transmitters, which choose the beamforming strategies, serve as players, and the utility function of each player is the achievable rate, which is the (average, where appropriate) mutual information. So, the set of feasible payoffs is $\mathcal{R}$, unless we allow scheduling, in which case it is $\overline{\mathcal{R}}$.

There are several possible choices for the disagreement point $\boldsymbol{\delta}$. The simplest is to let $\boldsymbol{\delta}=0$, which tacitly assumes that if the bargaining process fails, the network simply shuts down. Another common choice [32] is the security level of each player, or the maximum payoff a player can guarantee for itself even if other players conspire against it:

$$
\delta_{i}=\max _{\mathbf{w}_{i}} \min _{\mathbf{w}_{j}, j \neq i} I_{i}(\mathbf{W}) .
$$


In this case, each player pessimistically assumes only the worst-case rate should bargaining fail. Finally, we can choose the noncooperative Nash equilibrium rate as described in Section 2.4. Here we assume that if bargaining fails, players will simply act out of self-interest. Primarily due to simplicity, we take $\delta=0$ for the remainder of the paper. It is possible to modify our methods to accommodate an arbitrary $\boldsymbol{\delta}$, but only at the cost of increased computational complexity.

With the problem recast as a bargaining game, we can start looking for the K-S solution as defined in the previous section. Of course, in addition to finding the rates associated with the K-S solution, we need to find the beamformers (and, where appropriate, scheduling vector) that achieve the K-S rates. In this section we present algorithms that find the K$\mathrm{S}$ solution by constructing the rate-achieving beamformers and scheduling vector.

4.1. Without Scheduling. First we consider the problem without scheduling, in which case we can find the optimal $\mathrm{K}-\mathrm{S}$ beamformers. The first step is to find $\mathbf{b}$, the vector of best-case rates for each user. Fortunately, the best-case rates are easily computed. The best possible scenario for the $i$ th transmitter is when all other transmitters shut down, and the $i$ th transmitter uses the Nash equilibrium beamformer $\mathbf{w}_{i}^{\mathrm{NE}}=\mathbf{h}_{i, i} /\left\|\mathbf{h}_{i, i}\right\|$, giving a best-case rate of

$$
\begin{aligned}
b_{i} & =\log _{2}\left(1+\left|\frac{\mathbf{h}_{i, i}^{H} \mathbf{h}_{i, i}}{\left\|\mathbf{h}_{i, i}\right\|_{2}}\right|^{2}\right) \\
& =\log _{2}\left(1+\left\|\mathbf{h}_{i, i}\right\|_{2}^{2}\right) .
\end{aligned}
$$

Since we have chosen $\boldsymbol{\delta}=0$, the K-S solution forces the bargained rates $\mathbf{r}^{*}$ to lie along the line segment connecting the origin and $\mathbf{b}$. In other words, they must satisfy $\mathbf{r}^{*}=t \mathbf{b}$ for some scalar $0 \leq t \leq 1$. So, we can find the K-S rates and beamformers (which we gather into the matrix $\mathbf{W}$ ) by solving the following optimization problem:

$$
\begin{gathered}
\max _{\substack{t \in \mathbb{R} \\
\mathbf{W} \in \mathbb{C}^{N \times K}}} t \\
\text { subject to } t b_{i}=I_{i}(\mathbf{W}), \quad \forall i, \\
\left\|\mathbf{w}_{i}\right\|_{2} \leq 1, \quad \forall i .
\end{gathered}
$$

While the objective function and norm constraint in (16) are convex, the mutual information constraint is not. However, by slightly relaxing the problem, we can make the mutual information constraint convex. Instead of restricting ourselves to beamformers, we allow transmitters to choose covariance matrices $\mathbf{P}_{i}=E\left(\mathbf{x}_{i} \mathbf{x}_{i}^{H}\right)$ with arbitrary rank. We restrict the trace of the covariances to model the power constraint:

$$
\operatorname{tr}\left(\mathbf{P}_{i}\right) \leq 1, \quad \forall i,
$$

where $\operatorname{tr}(\cdot)$ denotes the matrix trace. In terms of covariances, the mutual information between $\mathbf{x}_{i}$ and $y_{i}$ is

$$
I\left(\mathbf{x}_{i} ; y_{i}\right)=\log _{2}\left(1+\frac{\mathbf{h}_{i, i}^{H} \mathbf{P}_{i} \mathbf{h}_{i, i}}{1+\sum_{j \neq i} \mathbf{h}_{i, j}^{H} \mathbf{P}_{j} \mathbf{h}_{i, j}}\right) .
$$

Exponentiating both sides and rearranging, the mutual information constraint can be written as

$$
\begin{gathered}
2^{r_{i}}=1+\frac{\mathbf{h}_{i, i}^{H} \mathbf{P}_{i} \mathbf{h}_{i, i}}{1+\sum_{j \neq i} \mathbf{h}_{i, j}^{H} \mathbf{P}_{j} \mathbf{h}_{i, j}}, \\
\mathbf{h}_{i, i}^{H} \mathbf{P}_{i} \mathbf{h}_{i, i}=\left(2^{r_{i}}-1\right)\left(1+\sum_{j \neq i} \mathbf{h}_{i, j}^{H} \mathbf{P}_{j} \mathbf{h}_{i, j}\right) .
\end{gathered}
$$

The equivalent constraint in (20) is affine (and therefore convex) with respect to the covariance matrices. Now, we can find the K-S solution as an optimization problem over the covariances:

$$
\begin{gathered}
\max _{\substack{\mathbf{P}_{i} \in \mathbb{C} \\
t \in \mathbb{R}}} t \\
\text { subject to } \mathbf{h}_{i, i}^{H} \mathbf{P}_{i} \mathbf{h}_{i, i}=\left(2^{t b_{i}}-1\right)\left(1+\sum_{j \neq i} \mathbf{h}_{i, j}^{H} \mathbf{P}_{j} \mathbf{h}_{i, j}\right), \quad \forall i . \\
\mathbf{P}_{i} \in S_{+}, \quad \forall i \\
\operatorname{tr}\left(\mathbf{P}_{i}\right) \leq 1, \quad \forall i
\end{gathered}
$$

where $S_{+}$is the set of positive semi-definite matrices. The mutual information constraint in (21) is convex with respect to the covariances but still nonconvex with respect to $t$. The structure of (21) allows a solution by iteratively using convex optimization techniques. Our approach is to choose $t$ according to the bisection method, using a convex feasibility test to see whether or not there exist feasible covariances that achieve the associated rates $\mathbf{r}=t \mathbf{b}$.

Given a fixed $t$, we test for feasibility by solving the following convex feasibility problem [33]:

$$
\begin{aligned}
& \text { find } \mathbf{P}_{1}, \ldots, \mathbf{P}_{K} \\
& \text { subject to } \mathbf{h}_{i, i}^{H} \mathbf{P}_{i} \mathbf{h}_{i, i}=\left(2^{t b_{i}}-1\right)\left(1+\sum_{j \neq i} \mathbf{h}_{i, j}^{H} \mathbf{P}_{j} \mathbf{h}_{i, j}\right), \\
& \qquad \mathbf{P}_{i} \in S_{+}, \quad \forall i \\
& \operatorname{tr}\left(\mathbf{P}_{i}\right) \leq 1, \quad \forall i .
\end{aligned}
$$

If the rates $\mathbf{r}=t \mathbf{b}$ are feasible, then performing the test in (22) also produces achieving covariance matrices. In our simulations, we test for feasibility using the convex programming package cvx [34].

We find the K-S covariances by combining the bisection line-search method with the feasibility test in (22), as depicted in Figure 4 . We start by setting $t^{\min }=0$ and $t^{\max }=$ 1. At iteration $k$, we choose the test point $t(k)$ defined by $t(k)=\left(t^{\max }+t^{\min }\right) / 2$. We then test the rate vector $\mathbf{r}(k)=$ $t(k) \mathbf{b}$ for feasibility by solving the problem defined by (22). If $\mathbf{r}(k)$ is feasible, then we set $t^{\text {min }}=t(k)$ and store the feasible covariances as the current solution. If $\mathbf{r}(k)$ is infeasible, we set $t^{\max }=t(k)$. Iterations continue until $t^{\max }-t^{\min }<\epsilon$ for small $\epsilon>0$. At this point, we choose the rates $\mathbf{r}^{*}=t^{\mathrm{min}} \mathbf{b}$, which are 


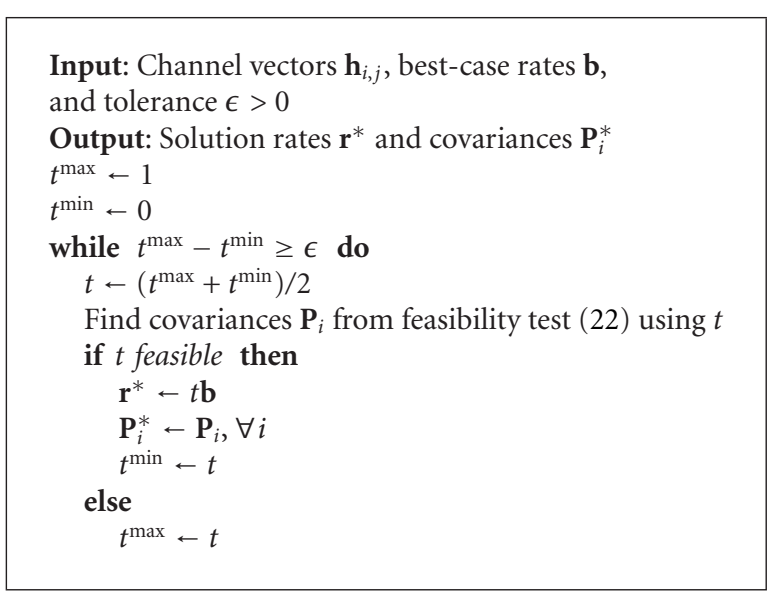

Algorithm 1: Kalai-Smorodinsky solution.

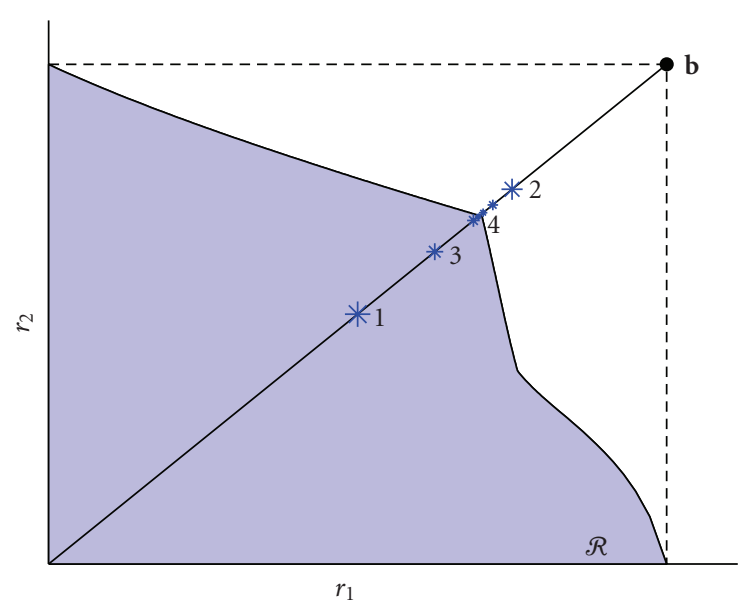

FIGURE 4: Depiction of bisection/feasibility algorithm for the K-S solution. The first few test points are numbered sequentially.

arbitrarily close to the K-S solution. We give a pseudocode summary of the procedure in Algorithm 1.

We emphasize that the generalization from beamformers to arbitrary-rank covariances is only an intermediate step that makes the feasibility problem convex. In [35] it is shown that any rates on the Pareto frontier (strong or weak) are achieved by rank-one covariances. Algorithm 1 therefore returns rank-one covariances except possibly for negligible numerical artifacts associated with the tolerance $\epsilon$. Experimentally, we indeed find that Algorithm 1 always returns rank-one covariances. The K-S beamformers are then easily extracted as the sole nontrivial eigenvector of each covariance matrix $\mathbf{P}_{i}^{*}$.

Finally, we can also adapt Algorithm 1 for an arbitrary disagreement point $\boldsymbol{\delta}$. The only real difficulty is to compute the best-case rates $\mathbf{b}$ for the new disagreement point. Fortunately, the bisection/feasibility test is easily adapted to compute $\mathbf{b}$. For each user $i$, we draw a line segment between $\boldsymbol{\delta}$ and the point $\mathbf{q}_{i}=\left(\delta_{1}, \ldots, \log _{2}\left(1+\left\|\mathbf{h}_{i, i}\right\|_{2}^{2}\right), \ldots, \delta_{K}\right)$. Using the bisection/feasibility method to find the maximal point on the line segment joining $\boldsymbol{\delta}$ and $\mathbf{q}_{i}$, we find the maximum rate $b_{i}$ for user $i$ such that every other user obtains the rates given in $\boldsymbol{\delta}$. Now we can straightforwardly adapt Algorithm 1 to find the K-S rates, which now lie on the line segment joining $\boldsymbol{\delta}$ and $\mathbf{b}$. However, the generality comes with a significant increase in complexity: since we have to run the bisection/feasibility algorithm for each user individually to find $\mathbf{b}$, the computational complexity is increased by a factor of $K$.

4.1.1. Asymptotic Performance. We start with a simple observation.

Proposition 1. Consider a fixed transmitter $j$ and set of receivers I that contains at least $N$ members, but $j \notin I$. If the vectors $\hat{\mathbf{h}}_{i, j}$ span all of $\mathbb{C}^{N}$, then the K-S rates $\mathbf{r}^{*} \rightarrow 0$ as $\rho_{i, j} \rightarrow \infty$ for all $i \in I$.

Proof. This result follows directly from the requirement $\mathbf{r}^{*}=$ $t$ b for scalar $0 \leq t \leq 1$. If one user's rate approaches zero, all rates must approach zero. We argue by contradiction. Supposing users' rates do not approach zero, $\left\|\mathbf{w}_{j}\right\| \geq d$ for some fixed $d>0$. But, since $\rho_{i, j} \rightarrow \infty$ for $i \in I$, the rates $r_{i}$ approach zero unless $\mathbf{w}_{j}$ is orthogonal to all $\hat{\mathbf{h}}_{i, j}, i \in I$. Since the vectors $\hat{\mathbf{h}}_{i, j}$ span $\mathbb{C}^{N}$, only $\mathbf{w}_{j}=0$ is orthogonal to them all, which is a contradiction.

The requirement that the vectors $\hat{\mathbf{h}}_{i, j}$ span $\mathbb{C}^{N}$ is mild, since most any generating distribution will produce linearly independent channel vectors almost surely until $\mathbb{C}^{N}$ is spanned. The condition $\rho_{i, j} \rightarrow \infty$ for fixed $j$ and several $i \in I$ is roughly equivalent to moving a cluster of receivers $i \in I$ closer and closer to transmitter $j$. ( Of course, the channel gains in a practical system will never approach infinity, but they can become large enough to induce the described asymptotic behavior.) While this scenario is somewhat unlikely, it represents a reasonable worst-case scenario. Similar statements hold when $K \rightarrow \infty$ and the gains $\rho_{i, j}$ are bounded away from zero, or when transmitter $j$ has inaccurate channel state information and $\rho_{i, j} \rightarrow \infty$ for any $i \neq j$. In a variety of asymptotic cases, the system responds to strong interference by simply shutting down.

It is perhaps unsurprising that rates go to zero when the interference gains $\rho_{i, j}$ or the number of users go to infinity. What is remarkable, however, is that all users' rates approach zero, even though only a subset of users needs to be shut down. This occurs because of the behavior of the K-S solution for nonconvex sets. The symmetry axiom precludes our shutting down some users but not others, and we are forced instead to accept the weakly Pareto efficient point $\mathbf{r}^{*}=0$. In Section 4.2, we show how the use of scheduling alleviates this drawback.

4.1.2. Pareto Efficiency. If we are willing to violate symmetry, we can extend the algorithm presented above to find (strongly) Pareto efficient rates that are at least as great as the K-S rates. After finding the K-S rates, we can randomly choose a user and use the bisection/feasibility method to increase the user's rate without decreasing other users' rates. 
More precisely, let $\mathbf{r}^{*}=\left(r_{1}^{*}, \ldots, r_{k}^{*}\right)$ be the $\mathrm{K}-\mathrm{S}$ rates, and randomly choose a user $i$. Then, we can test points along the line segment joining $\mathbf{r}^{*}$ and $\left(r_{1}^{*}, \ldots, b_{i}, \ldots, r_{k}^{*}\right)$ for feasibility as before. Thus, we maximize $r_{i}$ while keeping the other rates constant. After maximizing $r_{1}$, we can pick another user, maximize its rate, and continue until all users' rates are maximized. The resulting rates are strongly Pareto efficient by construction, but they no longer conform to the K-S axioms. In fact, they do not represent a bargaining solution in any sense: while they are at least as great as the K-S rates, they do not conform to any axioms other than Pareto efficiency.

Ensuring strong Pareto efficiency increases the computational burden by approximately a factor of $K$. In Section 5 , we explore the benefits obtained, showing that, except in asymptotic cases, the K-S solution produced by Algorithm 1 is typically close to a strongly Pareto solution.

4.2. With Scheduling. Using scheduling, the K-S solution is characterized by the beamformers and scheduling vector that maximize the objective function defined by the K-S solution:

$$
\begin{aligned}
J(\mathbf{W}(1), \ldots, \mathbf{W}(K), \mathbf{a}) & =\min _{i}\left(\frac{1}{b_{i}} \sum_{t=1}^{K} a_{t} I_{i}(\mathbf{W}(t))\right), \\
& =\min _{i}\left(\frac{r_{i}(S)}{b_{i}}\right),
\end{aligned}
$$

where we condense notation by collecting the beamformers and scheduling vector into a scheduling profile $S=$ $(\mathbf{W}(1), \ldots, \mathbf{W}(K), \mathbf{a})$ in the set $\delta=W_{1}^{K^{2}} \times \mathcal{A}$, and we let $r_{i}(S)=\sum_{t=1}^{K} a_{t} I_{i}(\mathbf{W}(t))$ denote user $i$ 's average rate.

Ironically, however, taking convex combinations of mutual information prevents us from transforming (23) into a series of convex problems as in Section 4.1. Instead, we seek a locally optimal solution, which suggests a gradientbased approach. Unfortunately, $J(S)$ is not continuously differentiable; in particular, the derivative is not continuous at the K-S point. So, instead of maximizing $J(S)$ directly, we successively maximize smooth approximations. Define

$$
F(S ; d)=\sum_{i=1}^{K} \ln \left(\frac{r_{i}(S)}{b_{i}}-d\right),
$$

with $d<\min _{i}\left(r_{i}(S) / b_{i}\right)$. Although it may not be immediately clear, we will see that maximizing $F(S ; d)$ is nearly equivalent to maximizing $J(S)$ for well-chosen $d$.

To maximize $F(S ; d)$ with respect to the beamformers and scheduling vector, we use the gradient projection method [36], a well-known method used to optimize a scalar function whose argument is an element of a convex set. It has been used to optimize similar multiantenna problems in [37-39].

First, we initialize the algorithm with a randomly chosen point $S^{0}=\left(\mathbf{W}^{0}(1), \ldots, \mathbf{W}^{0}(K), \mathbf{a}^{0}\right) \in \mathcal{X}$, and choose

$$
d^{0}=\min _{i}\left(\frac{r_{i}\left(S^{0}\right)}{b_{i}}\right)-\epsilon_{d}
$$

where $\epsilon_{d}>0$ is a small constant. That is, we set $d^{0}$ close to the minimum weighted average rate under $S^{0}$.
Next, we take a step in the direction of the gradient of $F\left(S^{0} ; d^{0}\right)$. The gradient with respect to the beamformers is found by first finding the gradient of each mutual information term $I_{i}(\mathbf{W}(t))$. Using the complex gradient $\nabla_{z} f(z)=\partial f(z) / \partial \Re(z)+j(\partial f(z) / \partial \Im(z))$, the gradient of the mutual information $I_{i}(\mathbf{W}(t))$ with respect to $\mathbf{w}_{j}(t)$ is

$$
\begin{aligned}
& \nabla_{\mathbf{w}_{j}(t)} I_{i}(\mathbf{W}(t)) \\
& = \begin{cases}\frac{2}{\ln 2}\left(\sigma_{i}(t)+v_{i}(t)\right)^{-1} \mathbf{h}_{i, j}^{H} \mathbf{w}_{j}(t) \mathbf{h}_{i, j}, & \text { for } i=j, \\
\frac{-2 \sigma_{i}(t)}{\ln 2}\left(\nu_{i}(t)\left(\sigma_{i}(t)+v_{i}(t)\right)\right)^{-1} \mathbf{h}_{i, j}^{H} \mathbf{w}_{j}(t) \mathbf{h}_{i, j}, & \text { for } i \neq j,\end{cases}
\end{aligned}
$$

where $\sigma_{i}(t)=\left|\mathbf{h}_{i, i}^{H} \mathbf{w}_{i}(t)\right|^{2}$ is the signal power at receiver $i$ during block $t$, and $v_{i}(t)=1+\sum_{j \neq i}\left|\mathbf{h}_{i, j}^{H} \mathbf{w}_{j}(t)\right|^{2}$ is the corresponding interference-plus-noise power.

Using the chain rule, the gradient of $F(S ; d)$ with respect to a beamformer $\mathbf{w}_{j}(t)$ is

$$
\nabla_{\mathbf{w}_{j}(t)} F(S ; d)=a_{t} \sum_{i=1}^{K}\left(\frac{r_{i}(S)}{b_{i}}-d\right)^{-1} \nabla_{\mathbf{w}_{j}(t)} I_{i}(\mathbf{W}(t)) .
$$

Since the scheduling vector is real-valued, the gradient with respect to $\mathbf{a}$ is simply a vector of partial derivatives:

$$
\nabla_{a_{t}} F(S ; d)=\sum_{i=1}^{K}\left(\frac{r_{i}(S)}{b_{i}}-d\right)^{-1} I_{i}(\mathbf{W}(t))
$$

Equations (28) and (29) highlight the connection between maximizing the sum of logs in $F(S ; d)$ and the minimum in $J(S)$. By setting $d$ close to the minimum weighted rate, $\left(r_{i}(S) / b_{i}-d\right)^{-1}$ becomes large for the minimumweighted-rate user $i$. So, the mutual information terms of user $i$ dominate the gradient of $F(S ; d)$, making it approximately proportional to the gradient of $J(S)$.

Having computed the gradient for each element of $S$, we take a step in the direction of steepest ascent:

$$
\widehat{S}^{0}=S^{0}+s \nabla_{S} F\left(S^{0} ; d^{0}\right),
$$

for fixed step size $s>0$. Theoretically, $s$ can be any constant [36], but since the factor $\left(r_{i}(S) / b_{i} /-d\right)^{-1}$ may be quite large, we take $s$ to be small, on the order of $\epsilon_{d}$. Of course, following the gradient may lead to an infeasible beamformer or scheduling vector. So, we project each $\hat{\mathbf{w}}_{i}^{k}(t)$ and $\hat{\mathbf{a}}^{k}$ onto the feasible sets $\mathcal{W}_{1}$ and $\mathcal{A}$, respectively. It is straightforward to show that the minimum-norm projections involve normalization and zeroing out, if necessary:

$$
\begin{gathered}
\operatorname{proj}_{W_{1}}\{\mathbf{w}\}= \begin{cases}\frac{\mathbf{w}}{\|\mathbf{w}\|}, & \text { for }\|\mathbf{w}\|>1, \\
\mathbf{w}, & \text { for }\|\mathbf{w}\| \leq 1,\end{cases} \\
\operatorname{proj}_{\mathcal{A}}\{\mathbf{a}\}=[\mathbf{a}-\lambda]^{+},
\end{gathered}
$$

where $[\cdot]^{+}=\max (\cdot, 0)$, and $\lambda \geq 0$ is a constant ensuring that the projected vector sums to unity. We can quickly solve for $\lambda$ 
using the bisection method. After taking a gradient step, we compute a new point $\widetilde{S}^{0} \in \delta$ defined by the projections onto the feasible space:

$$
\begin{gathered}
\tilde{\mathbf{w}}_{i}^{0}(t)=\operatorname{proj}_{w_{1}}\left\{\widehat{\mathbf{w}}_{i}^{0}(t)\right\}, \quad \forall i, t, \\
\widetilde{\mathbf{a}}^{0}=\operatorname{proj}_{\mathcal{A}}\left\{\hat{\mathbf{a}}^{0}\right\} .
\end{gathered}
$$

Finally, we choose a new point $S^{1}$ by stepping in the feasible direction defined by the projected vectors:

$$
S^{1}=S^{0}+\alpha^{0}\left(\widetilde{S}^{0}-S^{0}\right),
$$

for a variable step size $0 \leq \alpha^{0} \leq 1$. Since (33) defines a convex combination, we always have $S^{1} \in \S$. We choose $\alpha^{0}$ according to Armijo's rule along the feasible direction, which sets $\alpha^{0}=$ $\gamma^{m_{0}}$ for some $0 \leq \gamma \leq 1$ and $m_{0}$ the smallest nonnegative integer such that

$$
\begin{aligned}
& F\left(S^{1} ; d^{0}\right)-F\left(S^{1} ; d^{0}\right) \\
& \quad \geq \beta \gamma^{m_{0}}\left|\left\langle\nabla_{S} F\left(S^{0} ; d^{0}\right), \widetilde{S}^{0}-S^{0}\right\rangle\right| \\
& \quad=\beta \gamma^{m_{0}}\left|\left\langle\nabla_{\mathbf{a}} F, \widetilde{\mathbf{a}}^{0}-\mathbf{a}^{0}\right\rangle+\sum_{i, t}\left\langle\nabla_{\mathbf{w}_{i}(t)} F, \tilde{\mathbf{w}}_{i}^{0}(t)-\mathbf{w}_{i}^{0}(t)\right\rangle\right| .
\end{aligned}
$$

At the beginning of each subsequent iteration $k$, we choose $d^{k}$ by computing

$$
\left(d^{k}\right)^{\prime}=\min _{i}\left(\frac{r_{i}\left(S^{k}\right)}{b_{i}}\right)-\epsilon_{d} .
$$

If $\left(d^{k}\right)^{\prime}-d^{k-1}>\epsilon_{d}$ we choose $d^{k}=\left(d^{k}\right)^{\prime}$, and otherwise we choose $d^{k}=d^{k-1}$. Since Armijo's rule (34) ensures $F\left(S^{k} ; d^{k-1}\right)>F\left(S^{k-1} ; d^{k-1}\right)$, our choice of $d^{k}$ guarantees $d^{k}<$ $\min _{i}\left(r_{i}\left(S^{k}\right) / b_{i}\right)$, so $F\left(S^{k} ; d^{k}\right)$ is always well defined.

As before, we step in the direction of the gradient, but now using the function $F\left(S ; d^{k}\right)$, giving $\hat{S}^{k}=S^{k}+$ $s \nabla_{S} F\left(S^{k} ; d^{k}\right)$. We again take the projection $\widetilde{S}^{k}=\operatorname{proj}_{s} \hat{S}^{k}$ onto the feasible set, and we choose a new point according to the convex combination $S^{k+1}=S^{k}+\alpha^{k}\left(\widetilde{S}^{k}-S^{k}\right)$, with $\alpha^{k}$ decided by Armijo's rule. Iterations continue until

$$
\max \left|S^{k+1}-S^{k}\right|<\epsilon_{t}
$$

where $\max |\cdot|$ returns the absolute value of the maximal element of its argument. At convergence, the solution point $S^{*}=S^{k+1}$ is, within the specified tolerance, a stationary point of $F\left(S ; d_{k}\right)$. The algorithm is summarized in Algorithm 2.

Finally, we note that we cannot easily modify Algorithm 2 to use an arbitrary disagreement point $\boldsymbol{\delta}$. As before, the primary difficulty is computing the best-case rates $\mathbf{b}$ for the new disagreement point. Since Algorithm 2 operates on gradient ascent, we can only approximate the best-case rates. Since the best-case rates are so easily computed for $\boldsymbol{\delta}=0$, we focus exclusively on this case.

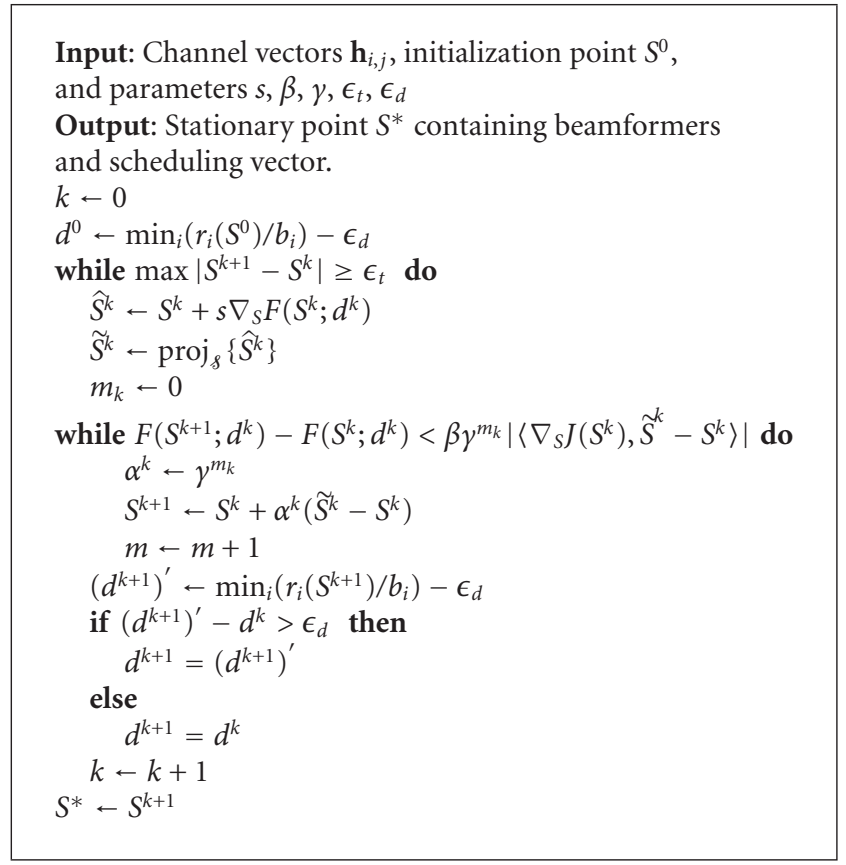

Algorithm 2: Kalai-Smorodinsky solution (with scheduling).

4.2.1. Convergence. The convergence of Algorithm 2 is guaranteed by the convergence of the sequence $\left\{d_{k}\right\}$. Since $b_{i}$ is the best-case rate, the average rate $r_{i}\left(S^{k}\right)$ cannot exceed $b_{i}$. Then, by definition, $d_{k} \leq \min _{i}\left(r(\mathbf{x})_{i} / b_{i}\right) \leq 1$ for all $k$. The sequence $\left\{d_{k}\right\}$ is therefore bounded, and since it is also nondecreasing, it must converge to a limit. Furthermore, since $d_{k}$ must increase by at least $\epsilon_{t}$ or remain constant, $\left\{d_{k}\right\}$ reaches its limit at finite $k$. Therefore, after a finite number of iterations, we perform gradient projection on $F(S ; d)$ for fixed $d$, which converges to a stationary point.

Of course, convergence to a stationary point of $F(S ; d)$ does not guarantee a good approximation to the K-S solution. Indeed, the result of Algorithm 2 does not, in general, satisfy the K-S axioms described in Section 3. However, if the solution point well-approximates the K-S point, then it may approximate the desirable properties of the K-S solution. So, we examine the solution point $S^{*}$ in terms of the criterion for the maximum of $J(S)$ : maximizing the minimum weighted rate.

By setting $d_{k}$ close to $\min _{i}\left(r_{i}\left(S^{k}\right) / b_{i}\right)$, we give priority to increasing the minimum weighted rate. Indeed, as we let $\epsilon_{d} \rightarrow 0$, the relative benefit of increasing the minimum weighted rate becomes arbitrarily large, suggesting that the algorithm will primarily focus on maximizing $\min _{i}\left(r_{i}\left(S^{k}\right) / b_{i}\right)$ until $r_{i}\left(S^{k}\right) / b_{i}=r_{j}\left(S^{k}\right) / b_{j}$ for all users. However, since $F(S ; d)$ is not convex, it is always possible for gradient projection to halt at a stationary point such that $r_{i}\left(S^{*}\right) / b_{i}$ and $r_{j}\left(S^{*}\right) / b_{j}$ are far apart. On the other hand, since we set $\epsilon_{d}$ to a fixed nonzero value, we can increase $F(S ; d)$ by increasing any one rate, even if we are at a stationary point for the minimum weighted rate. As a result, in practice, our algorithm tends to avoid such points, and $r_{i}\left(S^{*}\right) / b_{i}$ and $r_{j}\left(S^{*}\right) / b_{j}$ are close together. Since we cannot guarantee this 
analytically, in Section 5 we show by simulations that this is usually the case.

\section{Numerical Results}

5.1. Performance. To examine the performance of the proposed algorithms, we simulate on randomly generated channels. For our simulations, we choose $N=4$ and let $K$ vary. In each simulation, we randomly place $K$ transmitter/receiver pairs on the unit square. The channel coefficients are independently drawn from the zero-mean, unit-variance, complex Gaussian distribution. The channel gains $\rho_{i, j}$ are computed according to the path-loss model

$$
\rho_{i, j}=\frac{M}{d(i, j)^{\alpha}},
$$

where $d(i, j)$ is the Euclidean distance between the $j$ th transmitter and the $i$ th receiver, $M$ is an arbitrary constant, and $\alpha$ is the path loss exponent. In our simulations, we set $\alpha=4$ and choose $M=5 / 8$, which forces $\rho_{i, j}=10 \mathrm{~dB}$ when $d(i, j)=1 / 2$. For Algorithm 1 (and related methods), we set the convergence tolerance to $\epsilon=10^{-3}$. For Algorithm 2, we use parameters $s=10^{-3}, \epsilon_{d}=\epsilon_{t}=10^{-3}, \gamma=0.5$, and $\beta=0.05$.

In Figures 5 and 6 we examine algorithm performance in terms of efficiency and equity for $K=\{2,4,6,8,10\}$. We compare the proposed K-S algorithms with the max-min, max-sum, and TDMA rates. To compute the max-min rates, we modify Algorithm 1 to find the maximal rates such that all rates are equal. To maximize the sum rate, we employ a gradient-based method similar to [37], which returns a stationary point of the sum rate. The TDMA rates, computed easily by using the beamforming schedule from (11) provide a baseline for the scheduled K-S solutions. By definition, the TDMA rate for user $i$ is $b_{i} / K$. So, the rates satisfy $r_{i} / b_{i}=r_{j} / b_{j}$, making them the optimal scheduling of single-user rates in the K-S sense.

Figure 5 shows the average mutual information per user, averaged over 100 realizations for each value of $K$. Not surprisingly, the average rate is highest under sum rate maximization. Both K-S approaches degrade as we increase the number of users, but eventually the scheduling approach gives a better average rate in spite of the fact that it gives only a stationary point. In Figure 6 we examine the minimum mutual information across all links, averaged over the same 100 realizations. Max-min (again unsurprisingly) gives the highest minimum rate, followed by the K-S approaches. Max-sum gives the worst minimum rate, which drops nearly to zero beyond $K=2$. The $\mathrm{K}-\mathrm{S}$ solution allows us to maintain the sum rate while still protecting the weakest links.

Next, we focus on the performance of the scheduled K-S approach. Specifically, we examine how well the algorithm maintains the K-S constraint $r_{i} / b_{i}=r_{j} / b_{j}$. For each simulation, we compute the minimum normalized rate $c_{\text {min }}=\min _{i} r_{i} / b_{i}$. In Figure 7 , we plot the empirical cumulative distribution function (CDF) the deviation of the normalized rates from $c_{\min }$ for several values of $K$. An ideal CDF would form a sharp corner, meaning that all of the

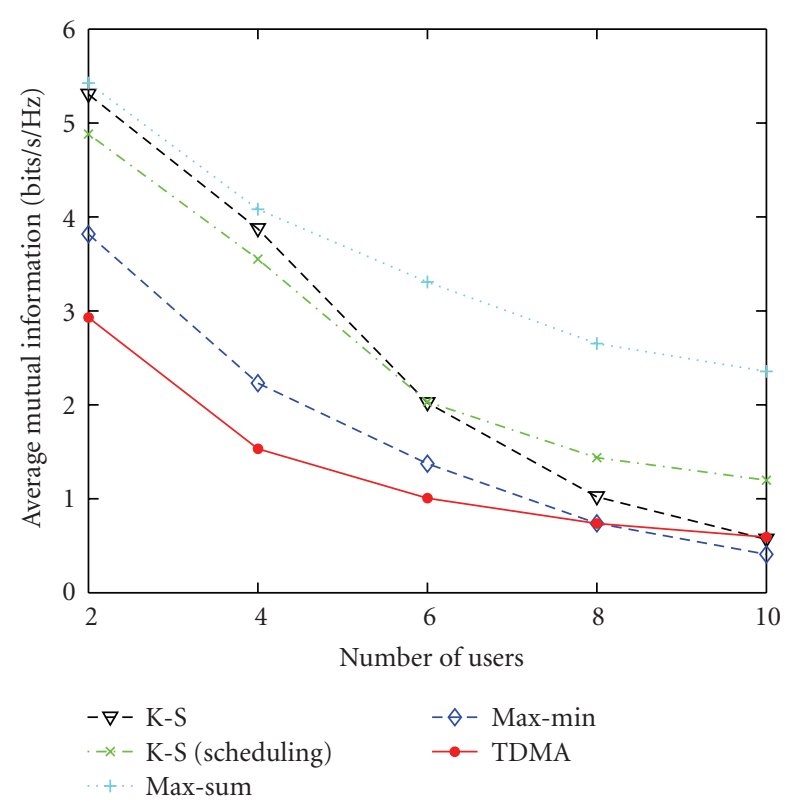

FIgURe 5: Average mutual information per user.

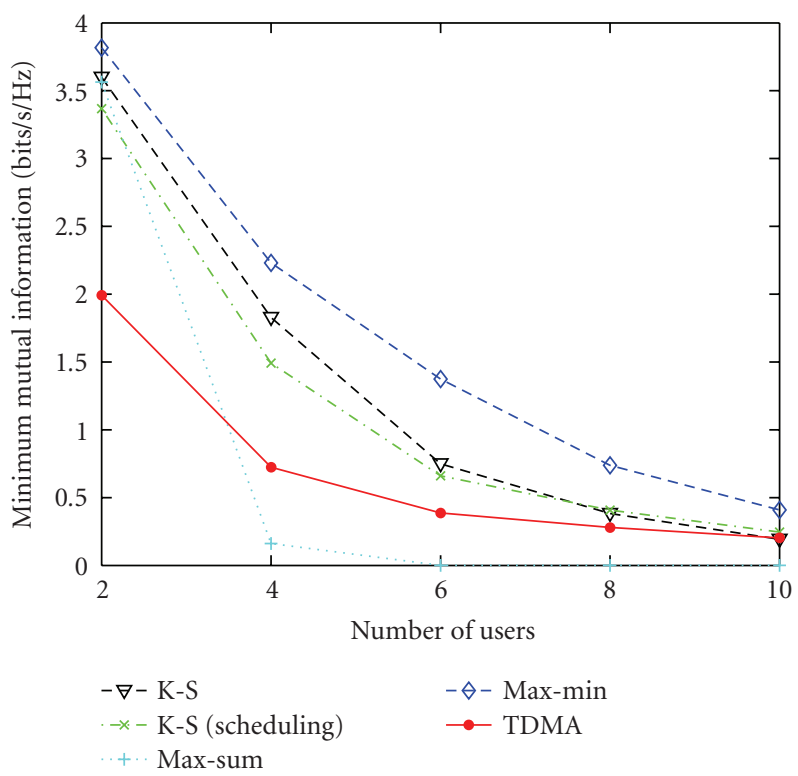

Figure 6: Average mutual information of the worst-case user.

deviations from $c_{\min }$ would be zero. The CDF for $K=2$ approximates the ideal case, with large deviations extremely rare. As $K$ increases, the corner increasingly rounds off-the normalized rates diverge more and more from $c_{\min }$. However, even for $K=10$, most normalized rates are close to $c_{\min }$.

5.2. Pareto Efficiency. Recall that since $\mathcal{R}$ is nonconvex, the K-S rates found by Algorithm 1 may be only weakly Pareto efficient. So, we compare the K-S rates to the strongly Pareto rates found in Section 4.1.2 to determine how often and how severely weakly Pareto rates occur. We set $N=3$ and $K=5$, and let $\rho_{i j}$ (in $\mathrm{dB}$ ) be uniformly distributed on the interval 


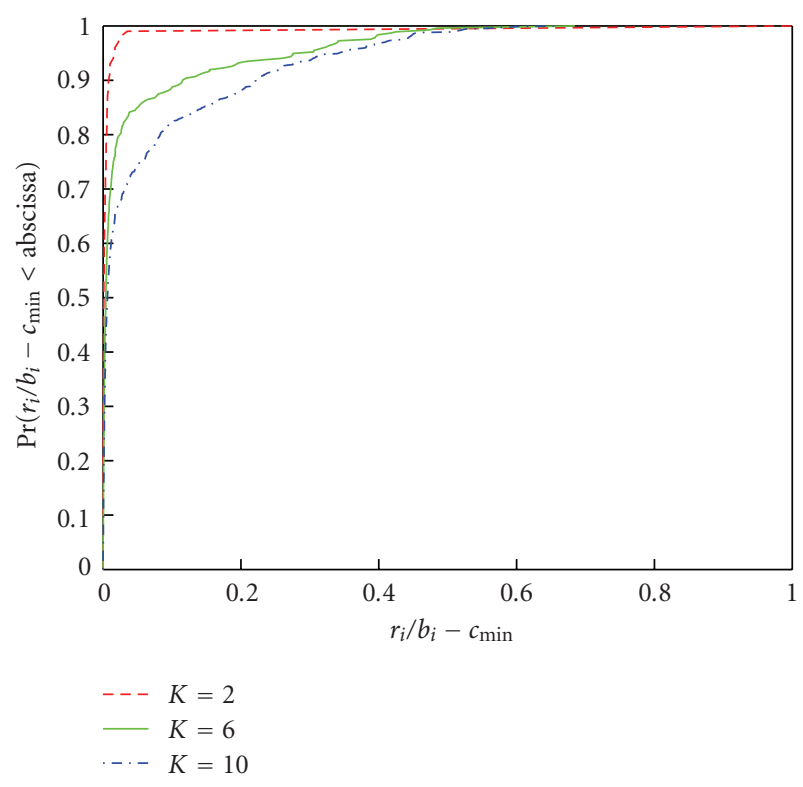

FIGURE 7: Empirical CDF showing the performance of Algorithm 2.

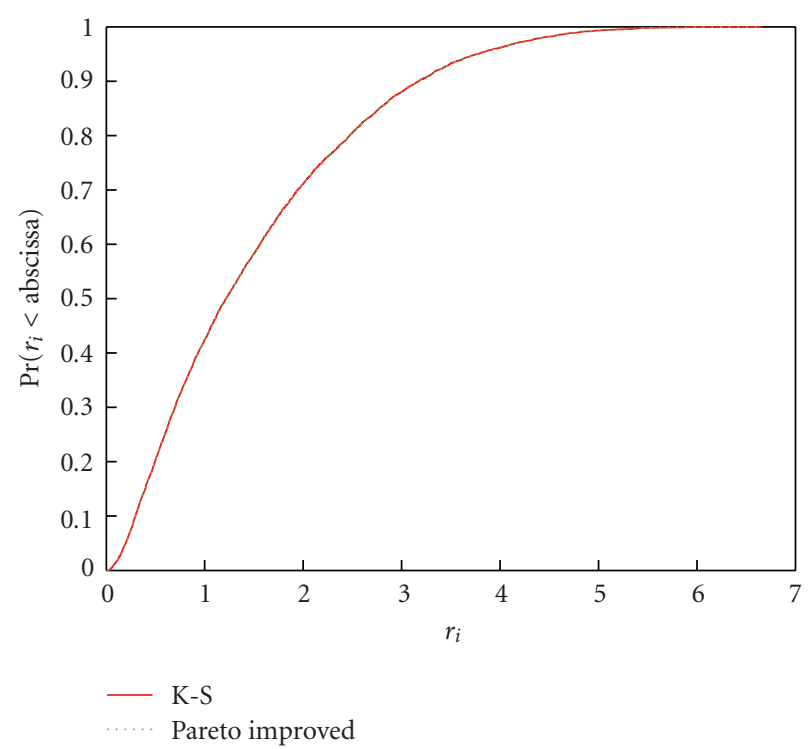

FIGURE 8: Empirical CDF: K-S rates versus Pareto improved rates.

$[5,30]$. In Figure 8 we show the CDF of the K-S and strongly Pareto efficient rates of 1000 independent realizations. The curves are essentially indistinguishable, showing that the K-S rates are strongly Pareto efficient in the vast majority of cases. While it is possible to find small-scale improvements, the difference is negligible on the whole, making the extension of Section 4.1.2 largely unnecessary.

5.3. Power Control. In [18] it is shown that, for a MISO interference channel with $K \leq N$, all strongly Pareto efficient rates can be achieved with unit-norm beamformers, making power control unnecessary. For $K>N$, however, we can

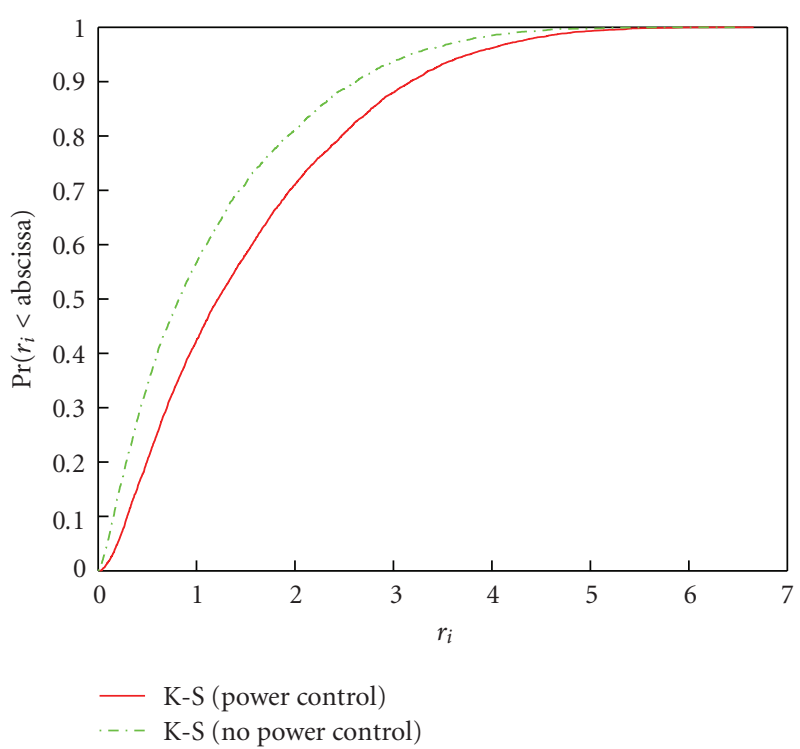

Figure 9: Empirical CDF: K-S rates versus fixed-power K-S rates.

easily find counter-examples in which strongly Pareto K-S rates are not achievable with unit-norm beamformers.

Since power control introduces additional complexity to a wireless system, we consider the loss associated with removing power control from the system. To do so, we slightly modify the K-S method presented in Section 4.1, changing the constraint $\operatorname{tr}\left(\mathbf{P}_{i}\right) \leq 1$ to a fixed-power constraint $\operatorname{tr}\left(\mathbf{P}_{i}\right)=1$ for all $i$. In Figure 9 we compare the $\mathrm{CDF}$ of the ordinary K-S rates with the fixed-power rates, using the same 1000 realizations from Section 5.2. Figure 9 shows a measurable loss: on average, users lose $24.7 \%$ of their total throughput by giving up power control.

\section{Conclusion}

We have proposed a method of beamformer selection for the MISO interference channel based on the Kalai-Smorodinsky bargaining solution from cooperative game theory. Using convex optimization techniques, we can efficiently find beamformers that achieve the K-S rates. Our numerical results demonstrate that despite the nonconvexity of $\mathcal{R}$, the $\mathrm{K}-\mathrm{S}$ solution is almost always strongly Pareto efficient for realistic signal-to-noise ratios. We have also shown that when $K>N$, power control is instrumental in achieving the K-S rates.

For cases of high interference, where $\mathcal{R}$ is highly nonconvex, we convexified the rate region by introducing scheduling, where transmitters may time-share among beamformers. We proposed a gradient-based method which approximates the K-S solution for this scenario. For sufficiently many users, the flexibility of time-sharing improves overall performance, even though it results in a local optimum. In both the convex and nonconvex approaches, the K-S bargaining provides a lower sum rate, but increased performance for weaker users, than maximizing the sum rate 
directly. Cooperative bargaining allows us to strike a balance between efficiency and equity for the interference channel.

\section{Acknowledgment}

This work has been partially supported by the US Army Research Office under Multi-University Research Initiative (MURI) Grant no. W91-1NF-04-1-0224.

\section{References}

[1] Y. Xiao, "IEEE 802.11n: enhancements for higher throughput in wireless LANs," IEEE Wireless Communications, vol. 12, no. 6, pp. 82-91, 2005.

[2] J. G. Andrews and A. Ghosh, Fundamentals of WiMAX: Understanding BroadbandWireless Networking, Prentice-Hall, Upper Saddle River, NJ, USA, 2007.

[3] D. Fundenberg and J. Tirole, Game Theory, MIT Press, Cambrige, Mass, USA, 2nd edition, 1991.

[4] V. Srinivasan, P. Nuggehalli, C. F. Chiasserini, and R. R. Rao, "Cooperation in wireless ad hoc networks," in Proceedings of the 22nd Annual Joint Conference on the IEEE Computer and Communications Societies (INFOCOM '03), vol. 2, pp. 808 817, San Francisco, Calif, USA, March-April 2003.

[5] Z. Han, Z. Ji, and K. J. R. Liu, "Fair multiuser channel allocation for OFDMA networks using Nash bargaining solutions and coalitions," IEEE Transactions on Communications, vol. 53, no. 8, pp. 1366-1376, 2005.

[6] R. A. Iltis, S.-J. Kim, and D. A. Hoang, "Noncooperative iterative MMSE beamforming algorithms for ad hoc networks," IEEE Transactions on Communications, vol. 54, no. 4, pp. 748 759, 2006.

[7] G. Arslan, M. F. Demirkol, and Y. Song, "Equilibrium efficiency improvement in MIMO interference systems: a decentralized stream control approach," IEEE Transactions on Wireless Communications, vol. 6, no. 8, pp. 2984-2993, 2007.

[8] J. Ellenbeck, C. Hartmann, and L. Berlemann, "Decentralized inter-cell interference coordination by autonomous spectral reuse decisions," in Proceedings of the 14th European Wireless Conference (EW '08), pp. 1-7, Prague, Czech Republic, June 2008.

[9] Y. Chen, H. T. Koon, S. Kishore, and J. Zhang, "Intercell interference management in WiMAX downlinks by a Stackelberg game between BSs," in Proceedings of the IEEE International Conference on Communications (ICC '08), pp. 3442-3446, Beijing, China, May 2008.

[10] G. Scutari, D. P. Palomar, and S. Barbarossa, "Competitive design of multiuser MIMO interference systems based on game theory: a unified framework," in Proceedings of the IEEE International Conference on Acoustics, Speech and Signal Processing (ICASSP '08), pp. 5376-5379, Las Vegas, Nev, USA, March-April 2008.

[11] M. T. Ivrlac and J. A. Nossek, "Intercell-interference in the Gaussian MISO broadcast channel," in Proceedings of the IEEE Global Telecommunications Conference (GLOBECOM '07), pp. 3195-3199, Washington, DC, USA, November 2007.

[12] M. Castaneda, A. Mezghani, and J. A. Nossek, "On maximizing the sum network MISO broadcast capacity," in Proceedings of the International ITG Workshop on Smart Antennas, (WSA '08), pp. 254-261, Vienna, Austria, February 2008.

[13] B. Song, Y.-H. Lin, and R. L. Cruz, "Weighted max-min fair beamforming, power control, and scheduling for a MISO downlink," IEEE Transactions on Wireless Communications, vol. 7, no. 2, pp. 464-469, 2008.

[14] E. G. Larsson and E. A. Jorswieck, "Competition versus cooperation on the MISO interference channel," IEEE Journal on Selected Areas in Communications, vol. 26, no. 7, pp. 10591069, 2008.

[15] E. A. Jorswieck and E. G. Larsson, "THE MISO interference channel from a game-theoretic perspective: a combination of selfishness and altruism achieves Pareto optimality," in Proceedings of the IEEE International Conference on Acoustics, Speech and Signal Processing (ICASSP '08), pp. 5364-5367, Las Vegas, Nev, USA, March-April 2008.

[16] E. Kalai and M. Smorodinksy, "Other solutions to Nash's bargaining problem," Econometrica, vol. 43, no. 3, pp. 513$518,1975$.

[17] J. F. Nash, “The bargaining problem,” Econometrica, vol. 18, no. 2, pp. 155-162, 1950.

[18] E. A. Jorswieck, E. G. Larsson, and D. Danev, "Complete characterization of the pareto boundary for the MISO interference channel," IEEE Transactions on Signal Processing, vol. 56, no. 10, part 2, pp. 5292-5296, 2008.

[19] J. F. Nash, "Non-cooperative games," Annals of Mathematics, vol. 54, no. 2, pp. 286-295, 1951.

[20] H. Peters and S. Tijs, "Individually monotonic bargaining solutions for $n$-person bargaining games," Methods of Operations Research, vol. 51, pp. 377-384, 1984.

[21] R. C. Douven and J. C. Engwerda, "Properties of $n$-person axiomatic bargaining solutions if the Pareto frontier is twice differentiable and strictly concave," Discussion Papers 50, Tilburg University Center for Economic Research, Tilburg, The Netherlands, 1995.

[22] A. E. Roth, "An impossibility result concerning $n$-person bargaining games," International Journal of Game Theory, vol. 8, no. 3, pp. 129-132, 1979.

[23] W. Thomson, "Two characterizations of the Raiffa solution," Economics Letters, vol. 6, no. 3, pp. 225-231, 1980.

[24] D. Bertsekas and R. Gallager, Data Networks, Prentice-Hall, Upper Saddle River, NJ, USA, 1987.

[25] E. L. Hahne, "Round-robin scheduling for max-min fairness in data networks," IEEE Journal on Selected Areas in Communications, vol. 9, no. 7, pp. 1024-1039, 1991.

[26] J. Rawls, A Theory of Justice, Belknap, Cambridge, Mass, USA, 1971.

[27] E. Kalai, "Proportional solutions to bargaining situations: interpersonal utility comparisons," Econometrica, vol. 45, no. 7, pp. 1623-1630, 1977.

[28] F. P. Kelly, A. K. Maulloo, and D. K. H. Tan, "Rate control for communication networks: shadow prices, proportional fairness and stability," Journal of the Operational Research Society, vol. 49, no. 3, pp. 237-252, 1998.

[29] A. Ibing and H. Boche, "Fairness vs. efficiency: comparison of game theoretic criteria for OFDMA scheduling," in Proceedings of the 41st Asilomar Conference on Signals, Systems and Computers (ACSSC '07), pp. 275-279, Pacific Grove, Calif, USA, November 2007.

[30] J. P. Conley and S. Wilkie, "The bargaining problem without convexity: extending the egalitarian and Kalai-Smorodinsky solutions," Economics Letters, vol. 36, no. 4, pp. 365-369, 1991.

[31] J. L. Hougaard and M. Tvede, "The Kalai-Smorodinsky solution: a generalization to nonconvex $n$-person bargaining," Discussion Papers 98-21, Department of Economics, University of Copenhagen, Copenhagen, Denmark, 1998.

[32] R. D. Luce and H. Raiffa, Games and Decisions, John Wiley \& Sons, New York, NY, USA, 1957. 
[33] S. Boyd and L. Vandenberghe, Convex Optimization, Cambridge University Press, Cambridge, UK, 2004.

[34] M. Grant and S. Boyd, "CVX: matlab software for disciplined convex programming," December 2008, http://www.stanford.edu/ boyd/cvx.

[35] X. Shang and B. Chen, "Achievable rate region for downlink beamforming in the presence of interference," in Proceedings of the 41st Asilomar Conference on Signals, Systems and Computers (ACSSC '07), pp. 1684-1688, Pacific Grove, Calif, USA, November 2007.

[36] D. P. Bertsekas, Nonlinear Programming, Athena Scientific, Belmont, Mass, USA, 2nd edition, 1995.

[37] S. Ye and R. S. Blum, "Optimized signaling for MIMO interference systems with feedback," IEEE Transactions on Signal Processing, vol. 51, no. 11, pp. 2839-2848, 2003.

[38] Y. Rong and Y. Hua, "Optimal power schedule for distributed MIMO links," IEEE Transactions on Wireless Communications, vol. 7, no. 8, pp. 2896-2900, 2008.

[39] M. Nokleby, A. L. Swindlehurst, Y. Rong, and Y. Hua, "Cooperative power scheduling for wireless MIMO networks," in Proceedings of the IEEE Global Telecommunications Conference (GLOBECOM '07), pp. 2982-2986, Washington, DC, USA, November 2007. 